\title{
Téoros
}

Revue de recherche en tourisme

\section{Les communications dans la réussite d'un événement}

\section{Ghislaine Choquette}

Volume 5, numéro 2, juillet 1986

Tourisme, fêtes et événements

URI : https://id.erudit.org/iderudit/1080577ar

DOI : https://doi.org/10.7202/1080577ar

Aller au sommaire du numéro

Éditeur(s)

Université du Québec à Montréal

ISSN

0712-8657 (imprimé)

1923-2705 (numérique)

Découvrir la revue

Citer cet article

Choquette, G. (1986). Les communications dans la réussite d'un événement. Téoros, 5(2), 29-32. https://doi.org/10.7202/1080577ar d'utilisation que vous pouvez consulter en ligne.

https://apropos.erudit.org/fr/usagers/politique-dutilisation/ 


\title{
Les communications dans la réussite d'un événement
}

\author{
par Ghislaine Choquette*
}

L'aspect communication est un élément capital dans la réussite d'un évếnement populaire. On ne doit surnout pas le négliger ou le sous-estimer. Nous connaissons tous des organisateurs qui un jour ou l'autre ont mis sur pied un événement et ont essuyé un échec ou obtenu un succès mitigé. Dans plusieurs cas, le volet communication est en cause.

Outre l'information, la communication englobe les relations puliques/relations de presse, la promotion et la publicité aux niveaux interne (l'organisation en général) et externe (le grand public, les publics spécifiques, les médias, etc). Afin de gérer efficacement les communications, les organisateurs doivent porter une attention particulière à la sélection des intervenants en communication, à la stratégie mise en place et a la portion du budget allouéce aux communications.

\section{Les communications, un rôle prépondérant}

Un choix judicieux s'impose

Qu'ils soient bênévoles ou rémunếrés. qu'ils oeuvrent à temps plein ou à mitemps, qu'ils soient nombreux ou en nombre restreint, il importe de bien sélectionner les communicateurs qui ont un rôle important. En effet, ils doivent jouer le röle d'agents d'information, d'attachés de presse et relationnistes, de promoteurs et de publicitaires...

- Lorsque les communicateurs sont des bénévoles, comme cela se produit souvent dans les événements populaires à caractère local et régional, il faut veiller à choisir des personnes ayant manifestement des goûts, des aptitudes et des connaissances en communication (un ex-journaliste, un animateur, un publicitaire, etc.). Il ne faut jamais confier ces responsabilités à quelqu'un qui ne s'y connait pas du tout. On peut trou-

\footnotetext{
"Ghislaine Choqueme agit en tant que consellere et agente de communication dans les secteurs public. parapubliç et privet depuis plus de 10 ans.

Elle est l'auteure d"un document de formation sur les communications, Les Communications et la Féte. toine par la Socibte des festivals populaires du Québec.
}

ver des bénévoles expérimentés et compétents mẻme dans la plus petite localité.

- Lorsque les organisateurs recourent aux services d'un(e) ou plusieurs professionnel(le)s, à contrat ou à la pige, ils doivent s'informer notamment des expériences antécédentes de l'employé(e), de ses forces principales, de sa disponibilité, de son esprit de création et de son sens de l'organisation. Toute entente doit se finaliser par un contrat ecrit en bonne et due forme (fonctions, tâches, délais, rémunération, modes de rémunération, etc.)

- Lorsque les organisateurs recourent, pour certaines tâches ou pour l'ensemble du dossier, à des firmes ou agences spécialisées en relations publiques, en promotion ou en publicité par exemple, il est recommandé de magasiner et de demander des soumissions écrites (au moins trois). La également, il faut finaliser toute entente par écrit.

Dans le cas des bénévoles, contractuels et pigistes, les communicateurs sont la plupart du temps regroupés au sein d'un comité, d'un service ou d'une direction de communication. Ils sont en général sous l'autorité du comité exécutif et/ou du conseil d'administration ou de la direction générale de l'événement et travaillent généralement dans les locaux de l'organisation.

\section{Des connaissances essentielles}

Les intervenants en communication doivent connaĭtre l'événement (objectifs, réalisations et principales étapes franchies), le milieu (histoiré, caractéristiques sociales. politiques et économiques) et les ressources humaines et techniques disponibles (organismes, associations, médias locaux et régionaux, bureaux régionaux des ministères, locaux et equipements, porte-parole. etc.). Bref, les communicateurs doivent savoir de quoi ils parlent, sur qui ils peuvent compter et de quoi ils peuvent disposer.

\section{De multiples fonctions}

Idéalement, lors de la première édition, on recourra aux services de spécialistes en communication/marketing afin d'analyser le marché et de créer l'identification de l'événement (symbole graphique, couleurs, typogaphie, etc.).

\section{Chaque année:}

- Les communicateurs doivent, en collaboration étroite avec les organisateurs. établir une stratégie de communication et voir à ce qu'elle soit respectée par l'organisation. Cette stratégie annuelle peut faire partie d'un plan quinquennal de développement de l'événement par exemple. Nous y reviendrons plus loin, compte tenu de son importance.

- Les communicateurs doivent voir à ce que I'identification de l'evenement soit intégrée partout (publications, objets promotionnels, publicité, affichage, etc.).

- Les communicateurs doivent être très vigilants en ce qui concerne l'image que l'événement projette ou désire projeter. Ils doivent donc être à l'affutt de la réaction des participants, des médias, des autorités, etc. Il reste que mëme les meilleurs communicateurs ne peuvent vendre très longtemps un événement mal défini, mal planifié et sans grand intérêt.

- Entre autres fonctions, les communicateurs doivent collaborer étroitement avec l'organisation interne (direction générale, autres comités, etc). Pour être au fait des derniers développements, il doivent assister aux reunions et rencontres (planification, programmation, évaluation, etc.).

- Le responsable des communications peut administrer le budget ou l'enveloppe budgétaire des communications evou travailler de très près avec le(s) responsable(s) des finances au sein de l'organisation,

- Il est souhaitable que le ou la responsable des communications fasse des recommandations entre autres sur le recrutement du personnel et des collaborateurs (bénévoles ou non) et le montant d'argent alloué aux communications. 


\section{Une stratégie de communication claire et efficace}

Les communicateurs qui pondent la stratégie peuvent être des consultants qui n'interviennent qu'à l'étape de la préparation du projet ou des bénévoles, contractuels, pigistes ou firmes qui interviendront également à l'étape de la réalisation. Cette dernière alternative est préférable.

Une stratégie claire et efficace ne peut se bắtir sans que les communicateurs aient été sensibilisés aux diverses facettes de l'événement (voir connaissances essentielles). Un des facteurs très importants est la somme $\mathrm{d}^{+}$argent que les organisateurs ont allouée ou prévoient allouer aux communications,

Les organisateurs doivent consacrer tout le temps nécessaire aux communicateurs et collaborer avec ouverture d'esprit et diligence afin que ceux-ci aient en main toutes les données. Autrement, il y aura perte d'énergies, de temps et d'argent.

La stratégie de communication doit être évidemment endossée par le conseil d'administration, la direction générale ou le ou la principal(e) responsable de l'événement selon le cas.

\section{Principales étapes}

- La détermination des objectifs

Les objectifs de communication sont axés, bien sûr, sur les objectifs généraux de l'événement déterminés par les organisateurs. Ainsi, si les organisateurs ont déterminé qu'un des principaux objectifs de l'événement 1986 est de susciter plus de participation des étudiants et des gens âgés, les communicateurs feront en sorte de rejoindre ces publics cibles par le biais de séances d'information, d'affiches, de lettres, d'émissions de radio spéciales, etc.

- Le choix des thèmes, sous-thèmes et slogans

Beaucoup d'organisateurs recourent à I'usage de thèmes, sous-themes et slogans. Ces véhicules de communication peuvent étre suggérés par la population, les bénévoles, les administrateurs, les communicateurs, etc. Peu importe les sources, les communicateurs doivent se sentir à l'aise avec les thèmes et slogans parce que ce sont eux qui ont à les véhiculer.

Dans le choix des thèmes, sous-thèmes et slogans, on se doit d'être créateurs. Ces vêhicules doivent coller à l'événement et correspondre aux objectifs généraux de l'événement et aux objectifs, spécifiques de l'année en cours. L'aide de professionnels peut êtré très utile à ce stade si les communicateurs sont des bénévoles par exemple qui ont une expérience limitée en communication.
- Le choix des opérations, des actions et des instruments

Tenant compte entre autres du marché. de l'envergure de l'événement. du bud. get disponible, du temps dont ils disposent et des ressources humaines et physiques sur lesquelles ils peuvent compter, les communicateurs doivent déterminer quelles seront les opérations à effectuer, les actions concrètes à poser et les instruments à utiliser.

Au niveau de l'information, ils pourront avoir recours entre autres aux communiqués de presse, à la conférence de presse, au bulletin paroissial, aux causeries et conférences ainsi qu'aux entrevues à la radio. L'organisation de soirées pour créer des liens entre les bénévoles, les réceptions spéciales pour la presse ou certains groupes, l'accueil des invités spéciaux, la mise sur pied de centres d'accueil et d'information sur le site sont autant d'éléments qui pourront être utilisés en relations publiques/relations de presse.

En promotion, une tournée de la mascotte et de la présidente d'honneur dans les régions voisines ainsi que la mise sur pied de kiosques itinérants en collaboration avec les commanditaires pourront être envisagés tandis qu'une série de messages à la radio et dans les journaux (quotidiens et hebdos) seront prévus en publicité.

- L'érablissement d'un cheminement critique et $d$ 'un echéancier

À l'instar des autres comités, services ou directions de l'événement, les communicateurs doivent déterminer où ils s'en vont. Fera-t-on la conférence de presse avant la tournée promotionnelle dans la région ou après, quand débutera la série de messages publicitaires à la radio et à la télévision, quand fera-t-on la distribution des programmes peuvent ètre autant de questions auxquelles il faut répondre à l'avance et de façon très précise.

Les communicateurs subdiviseront donc sur papier les tâches à accomplir en petites séquences et constitueront un calendrier où l'on retrouvera les dates précises de chaque séquence de travaux.

- Le contrôle, les ajustements et l'évaluation

Encore là, tout comme les autres comités. services ou directions et le conseil d'administration lui-même, les communicateurs doivent être vigilants et contröler l'efficacité de leurs opérations. Ils auront parfois à se réajuster très rapidement face à des situations imprévues telles une grève à la station de radio la plus importante de la région ou l'annulation d'un spectacle.
Finalement, les communicateurs auront a êvaluer l'ensemble des opérations effectuées et faire des recommandations aux organisateurs responsables de la tenue de l'événement. Cette dernière tâche est très importante et ne doit jamais ềre négligée mềne si elle peut hausser la facture au niveau des communications.

\section{Un budget adéquat et réaliste}

L'item communication est un poste budgétaire important. Habituellement, les organisateurs qui doivent budgéter longtemps à l'avance pour plusieurs raisons (demandes de subventions, études de faisabilité. etc.) optent pour un montant équivalent à $20 \%$ du budget global et réajustent au fur et à mesure.

Lorsque l'évếnement en est à sa première édition, le budget peut s'élaborer par comparaison avec d'autres événements de mémes type et envergure. Sinon, les budgets des éditions précédentes peuvent servir de guides. Lorsque les organisateurs ont des notions de communication ou l'experience des éditions précédentes, il peut être possible d'établir un budget assez réaliste.

Les communications engendrent beaucoup de dépenses mais peuvent générer également des recettes importantes (vente de publications et objets, apport des commandites, etc.). Les recettes sont plus difficiles à évaluer que les dépenses.

Il est souhaitable de détailler le plus possible toutes les dépenses et recettes prévisibles et de les budgeter séparément. Cette pratique facilite le contrôle et les réajustements qui doivent parfois s'effectuer très rapidement. Il faut penser aussi à certaines dépenses qui pourraient se poursuivre après la tenue de l'événement telles la distribution d'un dépliant promotionnel toute l'année durant et les déplacements des organisateurs invités à participer à certains évenements d'envergure.

Les prévisions budgétaires représentent des limites rationnelles. On ne doit pas les considérer comme des limites pouvant nuire au succès de l'opération des communications. Un budget se modifie, se révise, se repense et s'adapte. Il faut faire des prévisions réalistes. Il faut établir le plafonnement des dépenses et envisager des solutions de rechange (solutions moins coûteuses).

Puisque l'aspect monétaire est un facteur important dans l'élaboration de la stratégie de communication, il est souvent préférable que les organisateurs allouent aux communications une enveloppe budgétaire et que les communicateurs proposent une répartition de cette somme entre les éléments qu'ils jugent essentiels et les autres qu'on pourrait appeler de soutien. Ainsi, le programme et l'affiche peuvent être jugés éléments essentiels de la stratégie tandis que 
la tournée promotionnelle dans les régions voisines et l'album=souvenir sont considérés comme des éléments de soutien.

Dans ce cas, il arrive que les rémunérations des communicateurs soient incluses dans l'enveloppe budgétaire.

\section{Survol de certains éléments de la stratégie de communication}

De la causerie au vidéo, en passant par la circulaire et le reportage, nombreux sont les éléments qui peuvent faire partie de la stratégie. Voici un bref survol de certains de ces élléments.

\section{Le matériel}

Une part importante du budget de communication est affectée à la production et à la diffusion du matériel. Il faut donc dans un premier temps bien évaluer chaque année les besoins au niveau du matériel: publications, documents audiovisuels et objets en se posant des questions précises telles que: Imprimera-t-on un programme-souvenir en plus du programme? De combien de macarons a-t-on besoin? Un vidéo serait-il utile pour promouvoir l'événement?

Il faut ensuite en déterminer le contenu de base. A titre d'exemples: le programme contiendra la liste des activités, le logo, la photo du président d'honneur et les thèmes et sous-thèmes; le thème et le logo se retrouveront sur les banderoles, le film promotionnel contiendra essentiellement des entrevues avec les organisateurs, des bénévoles et les commanditaires impliqués dans l'événement.

Les étapes suivantes sont nombreuses et sont toutes importantes. On accordera beaucoup d'attention par exemple au choix des imprimeurs, des graphistes, des maisons spécialisées dans la vente d'objets promotionnels et des équipes de réalisation, à I'obtention de soumissions ćcrites, à l'établissement d'un calendrier de production et de diffusion et au suivi lors des étapes de production.

Souvent, les organisateurs recourent aux commandites et dons afin d'autofinancer en tout ou en partie la production du matériel. Il faut faire preuve de discernement et de bon goût lorsqu'il s'agit de déterminer la place ou l'espace accordé à ces individus. organismes, associations ou compagnies dont l'apport monétaire est souvent indispensable. Les ententes doivent être claires et respectées.

Selon l'envergure de l'événement et le nombre de documents produits, il peut être souhaitable qu'une personne soit responsable de la production et de la distribuition du matériel afin qu'aucune étape ne soit omise ou escamotée.

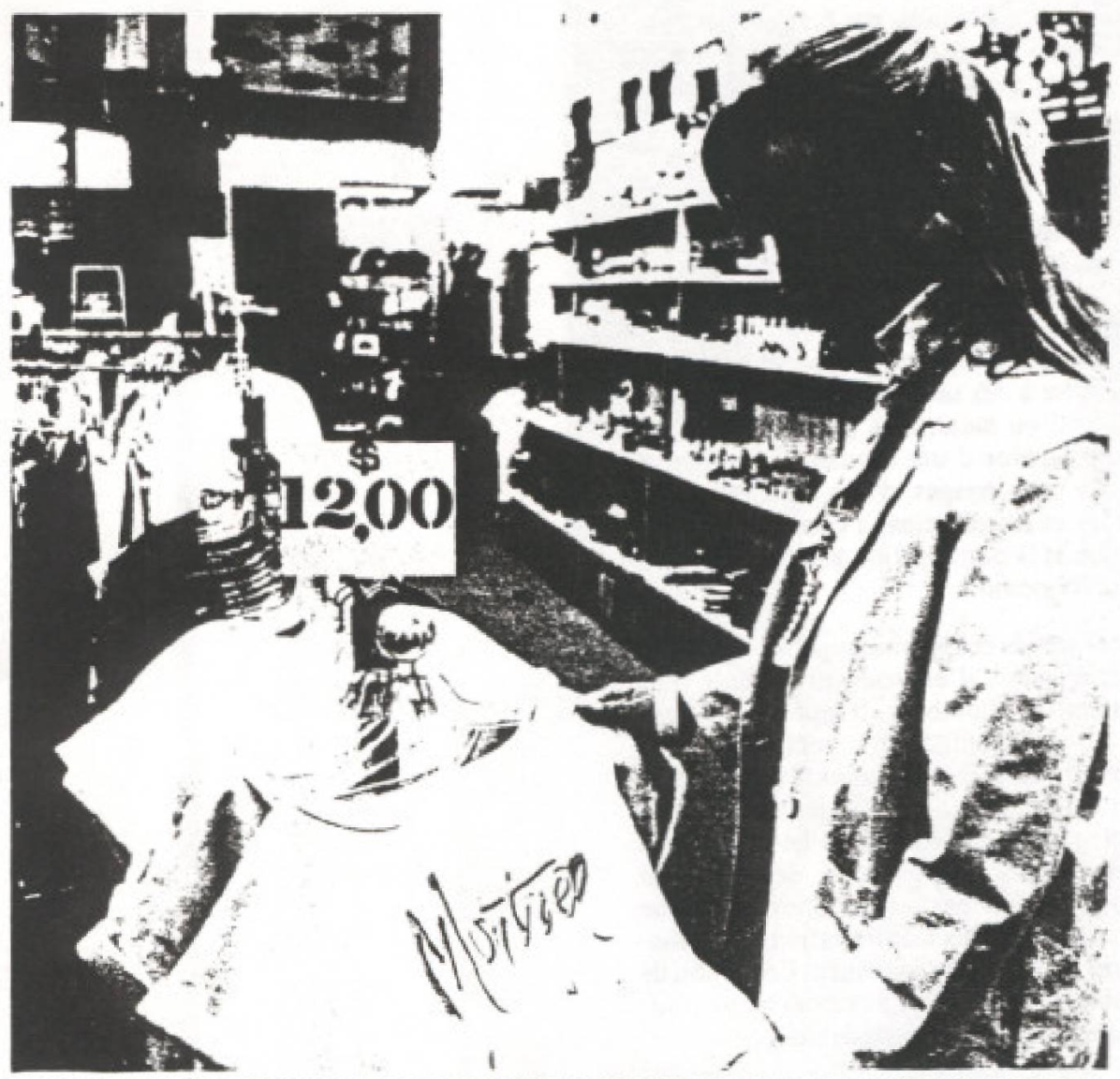

La production et la diffusion d'un bon matériel de support font partie de la stratégie de commu. nication.
Communiqués de presse, articles spetciaux, photos et dossiers d'information Le communiqué de presse est une nouvelle rédigée à l'intention des médias d'information qui peuvent le diffuser tel quel, le remanier ou le jeter au panier... L'élément indispensable est la nouvelle. Les communicateurs peuvent établir un calendrier de publication des communiqués en déterminant les sujets et le temps le plus propice à leur diffusion.

La rédaction du communiqué de presse fait appel à certaines techniques qu'il faut apprendre à maitriser. Plus le communiqué est bien écrit, bien présenté, adapté et d'intérêt immédiat pour les médias, plus il a de chances d'ètre diffusé. Il ne faut pas en abuser.

Les communicateurs peuvent aussi rédiger. à l'avance dans certains cas, des articles spéciaux (de fond) sur certains aspects de l'événement. A titre d'exemples, ces articles peuvent traiter de l'historique de l'événement, du président d'honneur et des artistes qui se produiront en spectacle.

Le contexte d'un événement populaire se prête bien à la photographie. On dit qu'une photo vaut 1000 mots. Les communicateurs peuvent utiliser les photos avant, pendant et après la tenue de l'événement accompagnées ou non de communiqués ou articles spéciaux.

Les photos doivent être de qualité. de préférence en noir et blanc (pour les médias écrits) et représenter des gens ou personnages en action si possible. Il est bon d'avoir une série de photos prêtes afin de satisfaire rapidement les demandes des médias. Il faut toujours joindre une légende adéquate à chacune des photos.

Plusieurs organisations préparent chaqué année des dossiers d'information sur l'événement. Ces dossiers peuvent être constitués de plusieurs éléments tels que: articles spéciaux, photos, illustrations, logo, dépliant promotionnel, revues de presse et informations sur les éditions précédentes, etc. Ces dossiers peuvent ềtre utilisés toute l'annce durant auprès de la presse mais peuvent servir à d'autres fins telle la recherche de commandite.

Conférences de presse et autres opérations destinées à la presse

La conférence de presse, tout comme le communiqué, doit comporter un élément nouveau qui est indispensable à sa réussite. Les organisateurs et communcateurs doivent l'utiliser quand ils jugent que c'est le meilleur moyen de transmettre une ou des nouvelle(s) intéressante(s) qui intéressent la presse maintenant.

L'organisation d'une conférence de presse implique beaucoup de préparatifs (on peut compter jusqu'à huit jours de travail) et 


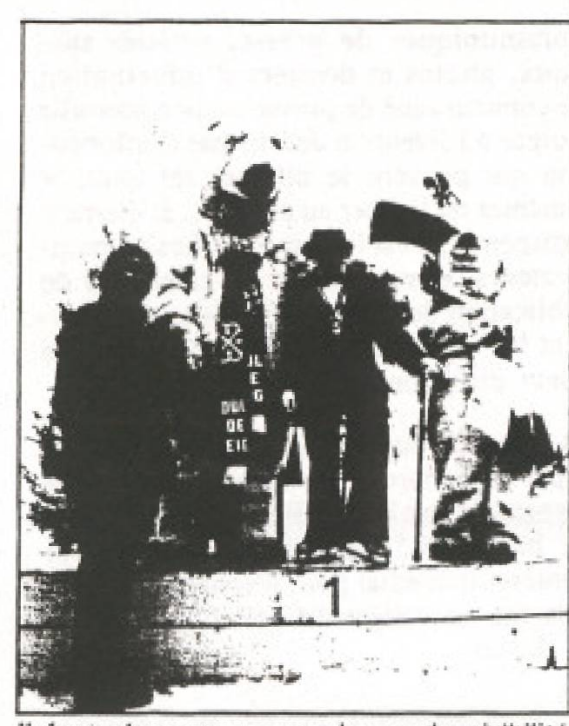

II faut s'assurer un maximum de visibilite d'une manifestation promotionnelle... et le photographe doit etre au rendez-vous.

entraîne des déboursés parfois importants. Lorsqu'elle est réussie, la conférence de presse apporte beaucoup de retombées. Le problème, c'est qu'on ne peut jamais être certain de sa réussite surtout dans les grands centres où les médias sont très sollicités et les événements nombreux.

D'autres événements tels que les diners et déjeuners de presse, des rencontres d'information et une visite organisée sur le site peuvent se substituer à la conférence de presse traditionnelle. Ces alternatives sont souvent moins coûteuses et la plupart du temps très efficaces. Ainsi, on peut inviter les chroniqueurs de tourisme et spectacles à un brunch où on leur remet entre autres un dossier-photos et convier des recherchistes à une rencontre d'information au local de l'organisation où on projettera un film sur l'evénement.

Qu'elles soient sollicitées par les communicateurs ou les médias d'information, les entrevues avec les médias sont un des instruments les plus efficaces pour répandre la nouvelle. Elles peuvent se faire en direct ou en différé et être utilisées avant, pendant et après l'événement. Elles exigent passablement de travail de la part des communicateurs qui doivent $s^{3} y$ prendre à l'avance surtout dans les grands centres et à la télévision.

Les porte-parole doivent être représentatifs et bien informés. Les médias (animateurs, recherchistes, journalistes, chroniqueurs, etc.) doivent recevoir de l'information adéquate avant l'entrevue. Des communicateurs habiles et expérimentés peuvent réussif à decrocher des entrevues dans des médias très variés et faire parler de l'événement sous plusieurs angles. On peut penser entre autres à une entrevue d'un artiste dans une émission de variétés à la télévision et à une entrevue de la présidente d'honneur dans un magazine destine aux femmes.

\section{Messages publicitaires}

La diffusion de messages publicitaires peut être très rentable surtout pour les evénements d'envergure régionale et nationale. Cependant, le domaine publicitaire est quelque peu complexe et les communicateurs qui s'y connaissent moins ont avantage a recourir aux services et/ou aux conseils de spécialistes ou du moins à transiger avec des médias en qui ils ont une parfaite confiance.

L'achat de temps d'antenne dans les médias écrits et électroniques entraine souvent des déboursés importants qui peuvent êttre réduits par l'apport de commandites et/ou des échanges avec les médias d'information. Plusieurs arrangements peuvent être conclus tels que: la station de radio diffusera le message deux fois plus souvent que le temps d'antenne payé en échange d'une identification de la station dans le programme, l'album-souvenir et sur les banderoles.

Le contenu des messages peut être élaboré par l'ćquipe des communications, par des consultants ou les médias qui ont généralement leurs propres services de concepteurs, de graphistes et de rédacteurs.

Dans les grands centres surtout, il est important de réserver à l'avance le temps d'antenne pour les messages à la radio, à la télévision et dans les médias spécialisés tels les magazines et revues. Les ententes doivent être finalisées par écrit et il faut toujours approuver (écouter et visualiser) les messages avant leur diffusion. Bien que la répétition soit souhaitable, il faut éviter la saturation.

\section{Manifestations et événements promo- tionnels \\ Les organisateurs et communicateurs peu- vent eux-mèmes mettre sur pied des mani- festions et/ou événements promotionnels ou répondre à des invitations au niveau local, régional ou national. Citons en exemples l'organisation d'une tournée de la mascote et des personnages symboliques dans les ecoles et les principaux commerces de la région et la participation à un salon touris- tique régional.}

Si ces sorties et apparitions peuvent ètre très intéressantes, il est souvent difficile d'en évaluer les retombées. Il faut donc s'assurer au maximum de leur rentabilité. Une façon d'augmenter la visibilité est d'y associer les médias en invitant par exemple un photographe à se rendre sur les lieux ou en participant à une émission de télévision lorsqu'on fait de la promotion dans une autre région. Ces manifestations et événements peuvent être également l'occasion de donner de la visibilité aux principaux commanditaires et/ou collaborateurs.

Dépendant de l'envergure de l'événement. certaines organisations recourent à ce type d'opérations promotionnelles plusieurs mois ou semaines avant la tenue de l'événement.

\section{En conclusion}

Les organisateurs qui veulent faire un succès de leur événement et veulent entre autres y attirer de plus en plus de touristes ne doivent pas improviser au niveau des communicarions et doivent s"entourer d'une solide équipe.

Ils se doivent également d'être ouverts aux témoignages, impressions et remarques des differents publics (résidents, touristes, commanditaires, médias, etc.).

Ils ne doivent pas avoir peur entre autres. de recourir aux enquêtes et sondages et doivent porter une attention spéciale à la revue de presse annuelle qui reflète l'opinion des médias et du grand public. $f$ 